\title{
Codon 8 (-AA) and Codons 22/23/24 (-AAGTTGG) Compound Heterozygous Deletion Mutation in the $\beta$-Globin Gene: The First Report in Turkey
}

\author{
Cemal Polat1 ${ }^{*}$, Osman Evliyaoğlu², Nuriye Mete³ ${ }^{3}$ Esra Türk4, Ahmet Yıldırım \\ ${ }^{1}$ Department of Biochemistry, Public Health Laboratuary, Kutahya, Turkey \\ ${ }^{2}$ Department of Biochemistry, Okmeydani Education and Research Hospital, Istanbul, Turkey \\ ${ }^{3}$ Department of Biochemistry, Faculty of Medicine, Dicle University, Diyarbakir, Turkey \\ ${ }^{4}$ Faculty of Medicine, Harran University, Sanliurfa, Turkey \\ ${ }^{5}$ Departmentof Pediatrics, Tekirdag State Hospital, Tekirdag, Turkey \\ Email: "drcpolat@gmail.com
}

Received 28 March 2016; accepted 12 April 2016; published 15 April 2016

Copyright (C) 2016 by authors and OALib.

This work is licensed under the Creative Commons Attribution International License (CC BY). http://creativecommons.org/licenses/by/4.0/

(c) $\underset{\mathrm{EY}}{\mathrm{B}}$ Open Access

\begin{abstract}
Background: Presentation of the first case of beta-thalassemia compound heterozygous with known mutations. Case Report: The patient was 3 years old girl. First symptoms were cough, fatigue, paleness. Hepatosplenomegaly were determined. Hematology parameters were: RBC 1.79 M/uL, Hb4.6 g/dL, Hct 12.3\%, MCV 68.7fL, MCH 25.7pg, RDW 31.5\%. The level of hemoglobin variants was: $\mathrm{HbF} 80.5 \%$, $\mathrm{HbA} 18.2 \%$, $\mathrm{HbA2} 1.3 \%$, and by molecular analysis codon $8(-\mathrm{AA})$ and codons 22/23/24(-AAGTTGG) compound heterozygous mutations were detected. Codon 8 (-AA) and codons 22/23/24(-AAGTTGG) heterozygous mutations were also detected in patient's mother and father respectively. Conclusion: Clinical manifestations such as late onset of symptoms and laboratory findings of patient with compound heterozygous deletion mutation were worse than homozygous patients whom are having the same mutations. It is important that the detection of carriers before the marriage to prevent the birth of patient children and genetic counseling is a good variety of ways of informing the public on the importance of prenatal diagnosis.
\end{abstract}

\section{Keywords}

Beta-Thalassemia, Compound Heterozygous Deletions, Codon 8 (-AA), Codons 22/23/24 (-AAGTTGG)

Subject Areas: Biochemistry, Genetics, Hematology, Pediatrics

\footnotetext{
${ }^{*}$ Corresponding author.

How to cite this paper: Polat, C., Evliyaoğlu, O., Mete, N., Türk, E. and Yıldırım, A. (2016) Codon 8 (-AA) and Codons 22/23/ 24 (-AAGTTGG) Compound Heterozygous Deletion Mutation in the B-Globin Gene: The First Report in Turkey. Open Access Library Journal, 3: e2536. http://dx.doi.org/10.4236/oalib.1102536
} 


\section{Introduction}

Beta $(\beta)$-thalassemia is an inherited disorder characterized by the inability to synthesize sufficient $\beta$-glob in chain which is controlled by the gene located on the short arm of chromosome 11. $\beta$-thalassemia is a single-gene disease and has autosomal recessive pattern, especially observed in the in the Mediterranean Region countries, characterized by hemolytic anemia and microcytosis. Patients with $\beta$-thalassemia require life-long blood transfusion and iron chelation therapy ensure enough hemoglobin $(\mathrm{Hb})$ level to prevent the accumulation of iron. While the rate of $\beta$-thalassemia carriers is $2 \%$ in overall country, but in some regions of Turkey ratio can be increase up to $10 \%$. In much of the population (in a geographic area or ethnic groups), more than $90 \%$ of cases have been found to be limited by mutation of 5 to 6 , which are the most commonly detected mutations such as IVS I-110 $(\mathrm{G} \rightarrow \mathrm{A})$, IVS I-6 (T $\rightarrow$ C), IVS II-745 (C $\rightarrow$ G), IVS I-1 (G $\rightarrow$ A), FSC-8 (-AA), IVS II-1 (G $\rightarrow$ A) [1]-[6].

In general, patients with $\beta$-thalassemia major have deletion mutations in a single gen, double-deletion mutation observed in our case. It was interesting observation the association of these mutations in Diyarbakır that was not found in the previous publications.

\section{Case Presentation}

A 3 years old girl admitted to our clinic in 05.02.2013 (Dicle University, Children Hospital, Department of Hematology), with complaint of cough, paleness, weakness. Hepatoslpenomegaly were determined by clinical examination. Complete blood count parameters were (Cell Dyn 3700, Abbott Laboratories, Abbott Park, IL, USA) RBC 1.79 M/uL, Hb 4.6 g/dL, Hct 12.3\% , MCV 68.7 fL, MCH 25.7 pg, RDW 31.5\%. Patient was followed up with the diagnosis of anemia. By the method of High Performance Liquid Chromatography, (Agilent 1100 series, Agilent Technologies, Waldbron, Germany) variants of Hb determined as HbF 80.5\%, HbA 18.2\%, HbA2 1.3\%. In the light of the follow-up $\beta$-thalassemia major diagnosed. Analysis of $\beta$-globin gene mutations was performed from whole blood samples, DNA was extracted and isolated from two pairs of primary (GML, Switzerland) then PCR (polymerase chain reaction) was performed. Sequence analysis of the gene determined with Big Dye ${ }^{\circledR}$ Terminator v3.1 Cycle Sequencing kit (Applied Biosystems, Inc., Foster City, CA, USA) and ABI PRISM 310 Genetic Analyzer (Applied Biosystems, Foster City, CA, USA). $\beta$-globinmutations were detected with SeqScape Software v2.6 program. Codon 8 (-AA) and codons 22/23/24 (-AAGTTGG) compound heterozygous mutations were detected (Figure 1). Codon 8 (-AA) heterozygous deletion was found in mother's sample and codons 22/23/24 (-AAGTTGG) heterozygous deletion mutation was found in father's sample (Figure 1). Verbal and written consent was obtained from the family.

\section{Discussion}

Large parts of $\beta$-thalassemia mutations are point mutations. Deletions are lesser extent [7]. $\beta$-thalassemia minor is a mild form of the disease. Individuals are heterozygous for the mutant allele. Morethan 200 mutations affecting the $\beta$-globin gene are now known to result in a phenotype of $\beta$-thalassemia. More than 40 of these mutations were reported in Turkey [8]-[10].

Patient's mother's mutation were at codon 8 (-AA) and father's mutation were at codons 22/23/24 (-AAGTTGG). Both of them were heterozygous mutations and diagnosed clinically as $\beta$-thalassemia minor compatible with the hematologic parameters (Table 1). $\beta$-thalassemia major is observed in patients with homozygous or compound heterozygous with a mutant $\beta$-globin allele. Codon 8 (-AA) deletion mutation is often seen in Turkey, but codons 22/23/24 (-AAGTTGG) deletion mutation is rare [3] [11]. Codon 8 (-AA) and codons 22/23/24 (-AAGTTGG) compound heterozygous mutations have not been reported in Turkey yet. Homozygous form of these two deletions was published in the literature which was presented clinically as $\beta$-thalassemia major but there was no case presented as $\beta$-thalassemia major which had been having compound heterozygous of above mutations. Hematology parameters of homozygous and heterozygous mutations of codon 8 (-AA) and codons 22/23/24 (-AAGTTGG) are shown in Table 2 [8] [12]. Codons 22/23/24 (-AAGTTGG) heterozygosity and Codon 8 (-AA) mutation heterozygosity showed mild presentation separately. In spite of the absence of HbA in homozygosity of Codon 8 (-AA) mutation, compound heterozygosity of these mutations has led to a more dramatic statement in $\mathrm{Hb}$ value.

$\beta$-thalassemia major is usually diagnosed during the first two years of birth [6]. Symptoms emerge during the second six months of life when gamma globin chain production decreases and is normally replaced with the 


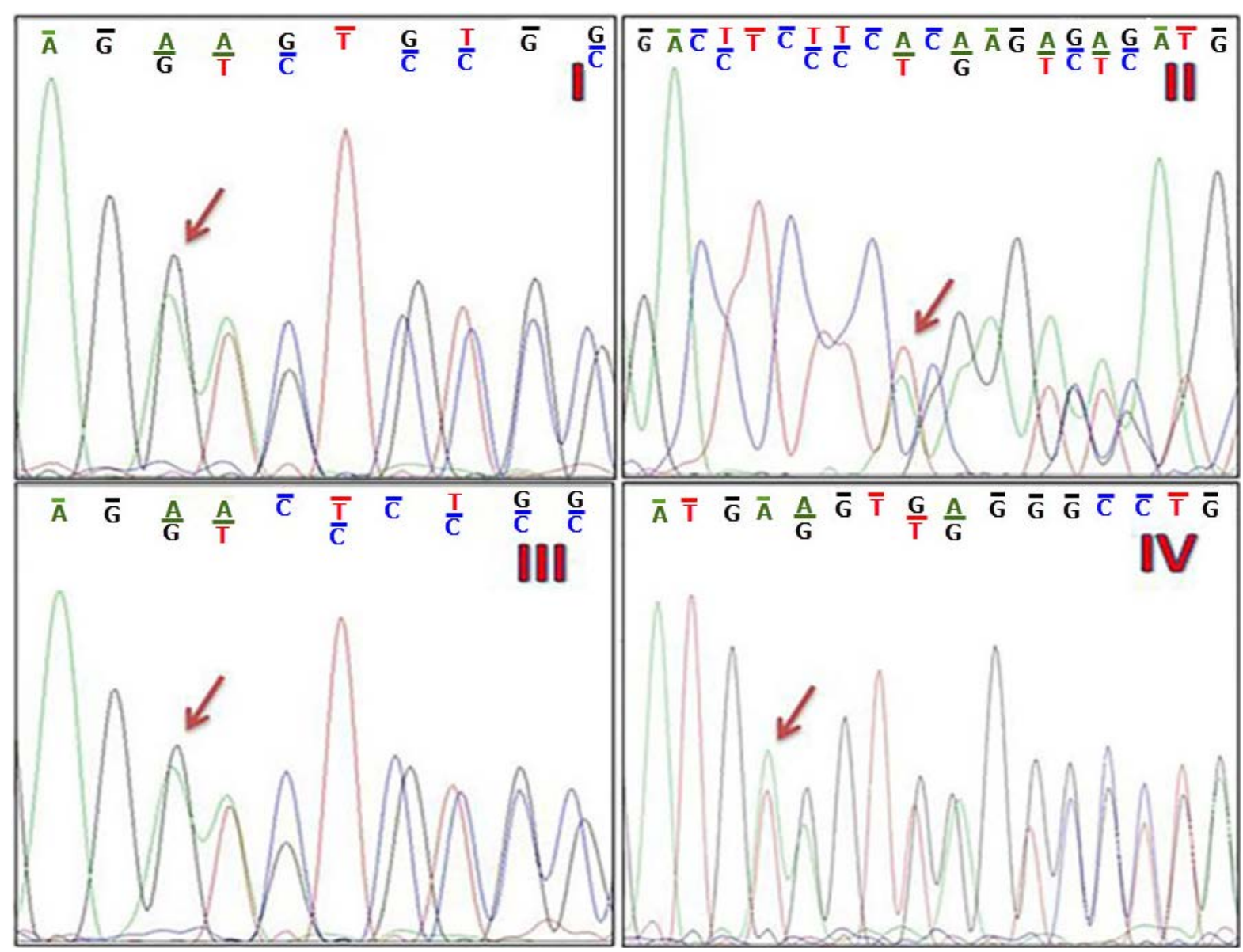

Figure 1. Deletions of $\beta$-globin genes. Patient [I $\rightarrow$ codon 8 (-AA), II $\rightarrow$ codons 22/23/24 (-AAGTTGG)], mother [III $\rightarrow$ codon 8(-AA)] and father [IV $\rightarrow$ codons22/23/24 (-AAGTTGG)].

Table 1. Hematologic parameters and hemoglobin variant levels of patient and parents.

\begin{tabular}{lccccccccc}
\hline & RBC (M/uL) & Hb (g/dL) & Hct (\%) & MCV (fL) & MCH (pg) & RDW (\%) & HbF (\%) & HbA (\%) & HbA2 (\%) \\
\hline Patient & 1.79 & 4.6 & 12.3 & 68.7 & 25.7 & 31.5 & 96.25 & 1.84 & 1.90 \\
Mother & 5.31 & 9.6 & 29.9 & 56.4 & 18.2 & 19.9 & 2.11 & 92.57 & 5.31 \\
Father & 6.22 & 12.1 & 37.1 & 59.7 & 19.5 & 19.0 & 2.28 & 92.03 & 5.68 \\
\hline
\end{tabular}

Table 2. Hematology parameters and values of $\mathrm{Hb}$ variants.

\begin{tabular}{|c|c|c|c|c|c|c|c|}
\hline & Mutation Type & Hb (g/dL) & $\operatorname{MCV}(\mathbf{f L})$ & MCH (pg) & HbA2 (\%) & HbF (\%) & HbA (\%) \\
\hline \multirow{2}{*}{ Codon 8 del } & Heterozygous [8] & $9.7-12.8$ & $68-74$ & $16.3-18.8$ & $4.1-5.1$ & $0.7-3.8$ & $91.1-95.2$ \\
\hline & Homozygous [8] & 10.6 & 82 & 29.4 & 1.4 & 98.6 & 0 \\
\hline \multirow{2}{*}{ Codons 22/23/24 del } & Heterozygous [12] & 10.3 & 64.8 & 24.9 & 5.4 & 3.1 & 92.5 \\
\hline & Homozygous & & & & & & \\
\hline $\begin{array}{c}\text { Codon } 8 \text { and Codons } \\
22 / 23 / 24 \text { del }\end{array}$ & Compound Heterozygous & 4.6 & 68.7 & 25.7 & 1.90 & 96.25 & 1.84 \\
\hline
\end{tabular}

8 and 12 are the reference numbers.

production of beta globin to form adult hemoglobin ( $\mathrm{Hb} \mathrm{A}$, alpha2/beta2). The clinical expression of the severe phenotype is remarkably heterogeneous, depending upon a variety of factors that alter the burden of alpha-globin inclusions in the individual patient. When $\beta$-thalassemia major becomes clinically apparent during the second 
six months of life, pallor, irritability, growth retardation, abdominal swelling due to hepatosplenomegaly, and jaundice reflect the onset and sequelae of severe hemolytic anemia. The symptoms associated with ineffective erythropoiesis (e.g., bony abnormalities and abnormal skeletal development) soon follow.

But clinically, this new compound heterozygous mutation became evident at three years old. This atypical presentation resulted in late diagnosis of the disease. It can be stated that compound heterozygous mutations impair the posttranscriptional or posttranslational regulation of hemoglobin chain more sophisticated manner than simple heterozygous mutations. The explanation of the delay in the clinic presentation of the disease needs more detailed investigations.

As consanguineous marriages in our country are very common and this culture increases the prevalence of the genetic diseases. In order to prevent the genetic diseases, it should be considered to detect the carriers before marriage, genetic counseling after marriage, informing the public on the importance of prenatal diagnosis.

\section{Conflict of Interest}

The authors declare no conflict of interest.

\section{Financial Disclosure}

In our study were excluded from the financial support.

\section{Poster Presentation}

Association of Clinical Biochemistry Professionals, International Participation Congress \& Lab Expo, 24-28 September 2013, Antalya.

\section{References}

[1] Tüzmen, S. and Schechter, A.N. (2001) Genetic Diseases of Hemoglobin: Diagnostic Methods for Elucidating $\beta$-Thalassemia Mutations. Blood Reviews, 15, 19-29. http://dx.doi.org/10.1054/blre.2001.0147

[2] Kazazian, H.H. and Boehm, C. (1988) Molecular Basis and Prenatal Diagnosis of $\beta$-Thalassemia. Blood, 72, 11071116.

[3] Altay, Ç. (2002) The Frequency and Distribution Pattern of $\beta$-Thalassemia Mutations in Turkey. Turkish Journal of Haematology, 19, 309-315.

[4] Chakrabarti, P., Gupta, R., Mishra, A., Rai, M., Singh, V.P. and Dash, D. (2005) Spectrum of $\beta$-Thalassemia Mutations in North Indian States: A $\beta$-Thalassemia Trait with Two Mutations in cis. Clinical Biochemistry, 38, 576-578. http://dx.doi.org/10.1016/j.clinbiochem.2005.03.002

[5] Thein, S.L. (1998) $\beta$-Thalassaemia. Baillière’s Clinical Haematology, 11, 91-126. http://dx.doi.org/10.1016/S0950-3536(98)80071-1

[6] Galanello, R. and Origa, R. (2010) $\beta$-Thalassemia. Orphanet Journal of Rare Diseases, 21, 5-11. http://dx.doi.org/10.1186/1750-1172-5-11

[7] Rund, D. and Rachmilewitz, E. (2001) Pathophysiology of $\alpha$ - and $\beta$-Thalassemia: Therapeutic Implications. Seminars in Hematology, 38, 343-349. http://dx.doi.org/10.1016/S0037-1963(01)90028-9

[8] (2013). http://globin.bx.psu.edu

[9] Tadmouri, G.O. and Başak, A.N. (2001) $\beta$-Thalassemia in Turkey: A Review of the Clinical, Epidemiological, Molecular and Evolutionary Aspects. Hemoglobin, 25, 227-234. http://dx.doi.org/10.1081/HEM-100104031

[10] Thein, S.L. (2005) Genetic Modifiers of $\beta$-Thalassemia. Haematologica, 90, 649-660.

[11] Ozçelik, H., Başak, A.N., Tüzmen, S., Kirdar, B. and Akar, N. (1993) A Novel Deletion in A Turkish $\beta$-Thalassemia Patient Detected by DGGE and Direct Sequencing: FSC22-24(-7bp). Hemoglobin, 17, 387-391. http://dx.doi.org/10.3109/03630269308997492

[12] Nadkarni, A., Gorakshakar, A., Surve, R., Sawant, P., Phanasgaonkar, S., Nair, S., et al. (2009) Hematological and Molecular Analysis of Novel and Rare $\beta$-Thalassemia Mutations in the IndianPopulation. Hemoglobin, 33, 59-65. http://dx.doi.org/10.1080/03630260802626012 